\title{
Rectennas at optical frequencies: How to analyze the response
}

\author{
Saumil Joshi ${ }^{\text {a) }}$ and Garret Moddel ${ }^{\text {b) }}$ \\ Department of Electrical, Computer, and Energy Engineering, University of Colorado, Boulder, \\ Colorado 80309-0425, USA
}

(Received 18 May 2015; accepted 16 August 2015; published online 31 August 2015)

\begin{abstract}
Optical rectennas, antenna-coupled diode rectifiers that receive optical-frequency electromagnetic radiation and convert it to DC output, have been proposed for use in harvesting electromagnetic radiation from a blackbody source. The operation of these devices is qualitatively different from that of lower-frequency rectennas, and their design requires a new approach. To that end, we present a method to determine the rectenna response to high frequency illumination. It combines classical circuit analysis with classical and quantum-based photon-assisted tunneling response of a high-speed diode. We demonstrate the method by calculating the rectenna response for low and high frequency monochromatic illumination, and for radiation from a blackbody source. Such a blackbody source can be a hot body generating waste heat, or radiation from the sun. C 2015 AIP Publishing LLC.

[http://dx.doi.org/10.1063/1.4929648]
\end{abstract}

\section{INTRODUCTION}

Recently, there has been a surge of interest in optical antennas connected to high-speed nonlinear diodes. Such systems, known as optical rectennas, have been investigated as optical and high-frequency detectors, ${ }^{1-5}$ and for infrared and visible-light-frequency energy-harvesting. ${ }^{6-10}$ They incorporate nano-antennas and high-speed diodes such as metal-insulator-metal and geometric diodes for high frequency rectification. ${ }^{11-20}$ However, no simple method exists for analyzing rectenna performance at optical frequencies. In this paper, we present a method to calculate the optical response and performance of a diode in an optical rectenna. The analysis presented here is different from the one in Ref. 21, which provides the fundamental physical concepts. Here, we apply those concepts to the electrical circuit theory of rectennas using equivalent electrical circuit analysis methods. The efficiency limits of rectennas have been developed using this approach in Ref. 9.

To determine the response of the rectenna under optical illumination, we apply the theory of photon-assisted tunneling (PAT) to high-speed diode operation, ${ }^{21,22}$ which cannot be explained using classical large-signal theory traditionally used for microwave rectenna analysis. ${ }^{23}$

The optical response of a rectenna depends on the performance of its components, which include an antenna, a diode, a low-pass filter, and a load, as shown in Figure 1. The antenna collects incident electromagnetic waves and generates an alternating current, which is rectified by a highspeed diode. A low-pass filter allows only rectified DC to flow to the load. The load current can be used for detection or energy harvesting of electromagnetic radiation. When used for energy harvesting, the performance of the rectenna is determined by calculating the rectenna efficiency, which is

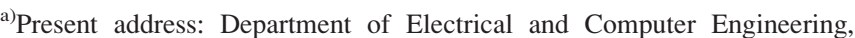
University of Massachusetts, Amherst, Massachusetts 01003, USA.

b) Author to whom correspondence should be addressed. Electronic mail: moddel@colorado.edu.
}

the ratio of the DC output power across the load $\left(P_{\text {out }}{ }^{D C}\right)$ and the AC input power

$$
\eta=\frac{P_{o u t}^{D C}}{P_{\text {in }}^{A C}} .
$$

The $P_{i n}{ }^{A C}$ is the AC power available at the antenna terminals, and is the product of the incident electromagnetic power density at the location of the antenna, its effective area, and efficiency.

\section{RECTENNA EQUIVALENT CIRCUIT}

As shown in Figure 2, the antenna is represented by a Thévenin-equivalent generator (an AC voltage source represented by $\left.v_{S}=V_{S} \cos (\omega t)\right)$ in series with the antenna input impedance, which at the resonant frequency is represented by the radiation resistance $\left(R_{S}\right)$. The Thévenin-equivalent voltage across the antenna is a function of the input power $\left(P_{i n}{ }^{A C}\right)$, and is calculated using the energy conservation principle as follows. In Figure 2(a), we show a simple equivalent circuit of an antenna connected to an impedance-matched resistive load. When illuminated by a monochromatic source of angular frequency $\omega$, maximum power transfer occurs between the antenna and the load, such that $P_{i n}{ }^{A C}$ is sent to the load without reflection. The peak value of the source voltage is calculated as

$$
V_{S}=\sqrt{8 R_{S} P_{i n}^{A C}}
$$

The optical rectenna can be modeled as the equivalent electrical circuit shown in Figure 2(b). A capacitor $C$ acts as the clamping capacitor for the rectenna circuit, in addition to modeling the fact that the antenna blocks DC. This results in an alternating voltage across the diode that is clamped by a $\mathrm{DC}$ voltage. In this way, the output DC voltage can rise to the AC peak voltage and, under ideal conditions, provide a rectification efficiency that approaches $100 \%$. The DC clamp voltage across the capacitor is the operating voltage $\left(V_{O}\right)$ 


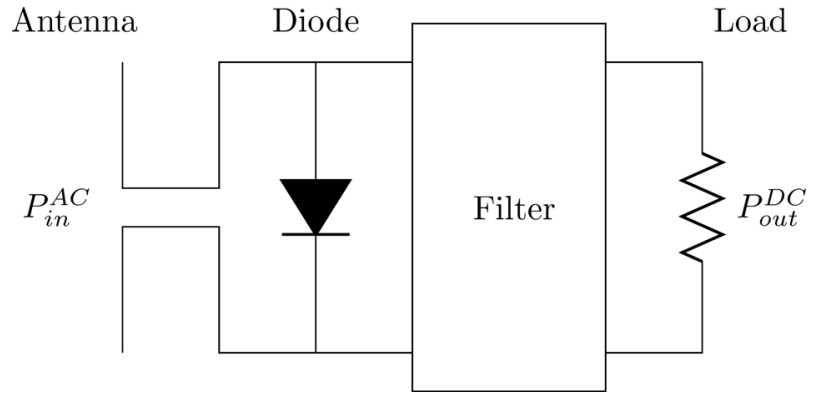

FIG. 1. General schematic of a rectenna. The input power available at the antenna is $P_{i n}{ }^{A C}$. The diode rectifies the AC signal to produce DC. The lowpass filter blocks the AC from being lost in the load. $P_{\text {out }}{ }^{D C}$ is the rectified DC output power provided to the load.

that appears across the load (previously referred to as $V_{D}$ (Ref. 21)). ${ }^{9}$ The load is connected in parallel with the diode through a low-pass filter $L$ to allow only DC to flow through the load and block the AC power from being dissipated in it. The $P_{\text {out }}{ }^{D C}$ is the product of the rectified DC output current, which we refer to as the illuminated DC $\left(I_{\text {illum }}\right)$, and the DC output voltage, which is the operating voltage of the rectenna.

In Secs. III-V, we demonstrate a method to determine the optical response of the rectenna by applying the theory of PAT to a diode in the rectenna equivalent circuit, unifying the theory of PAT with conventional circuit analysis. We plot the illuminated $I(V)$ characteristics, $I_{\text {illum }}$ vs. $V_{O}$, under monochromatic and broadband illumination conditions.

\section{PROCEDURE TO CALCULATE RECTENNA ILLUMINATED I(V) CHARACTERISTICS}

In this section, we present the procedure to calculate the illuminated $I(V)$ characteristics of the rectenna. Before analyzing the equivalent circuit with a resistive load to determine the illuminated $I(V)$ characteristics, we consider a simple case in which the load is a short circuit. This case illustrates and develops an understanding of how the (a)

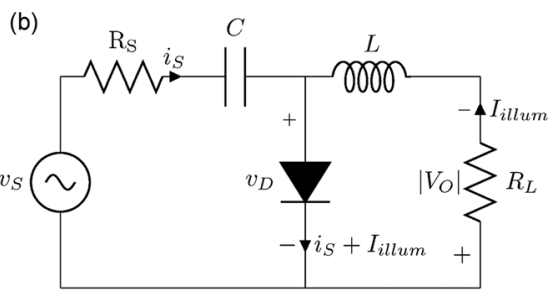

FIG. 2. Equivalent circuit representation of a rectenna. (a) Circuit representing an antenna connected to an impedance-matched resistive load. The antenna is represented by an AC voltage source $v_{S}$ connected to the antenna radiation resistance $R_{S}$, and the load $R_{L}$. The current flowing through the load is denoted by the arrow. Maximum power transfer occurs between the antenna and the load when $R_{S}=R_{L}$. (b) Rectenna equivalent electrical circuit showing the antenna source voltage $v_{S}$, and its equivalent radiation resistance $R_{S}$. A capacitor $C$ blocks the flow of DC in the antenna circuit, and also provides voltage clamping. The inductor $L$ forms a low-pass filter to block the AC power from being dissipated in the load. The AC in the antenna circuit is $i_{S}$ and the rectified DC is $I_{\text {illum }}$. The total (AC plus DC) voltage across the diode is $v_{D}$ and the total current through the diode is $i_{S}+I_{i l l u m}$. The load resistance is $R_{L}$ and the DC operating voltage across the load is $V_{O}{ }^{24}$ rectenna generates a DC illuminated short-circuit current and works as a detector. Because the load has zero resistance, the operating voltage, equal to the clamping voltage across $C$, is zero, which simplifies the equivalent circuit. Kirchhoff's voltage law applied to the antenna-diode loop of the circuit gives the instantaneous voltage across the diode

$$
v_{D}(t)=v_{S}(t)-i_{S}(t) R_{S}
$$

The short-circuit mode results in the circuit of Figure 2, but with $R_{L}$ replaced by a short. When a time-dependent voltage is applied to the diode, it produces an AC that flows through the antenna circuit, and a DC that flows as the shortcircuit current $\left(I_{S C}\right)$ through the diode-filter loop. The $I_{S C}$ is the point that intersects the current axis on the illuminated $I(V)$ characteristics of the rectenna.

When a non-zero resistance load is placed in the circuit, $I_{\text {illum }}$ flows through the load and the DC voltage is

$$
\left|V_{O}\right|=I_{\text {illum }}\left(V_{O}\right) R_{L}
$$

Since the $V_{O}$ also appears across the capacitor $C$ as the clamping voltage, the effect of the load resistance is to change the time-dependent voltage across the diode, which is now the sum of the operating voltage across the capacitor and an AC voltage

$$
v_{D}(t)=-\left|V_{O}\right|+v_{S}(t)-i_{S}(t) R_{S} .
$$

To calculate the illuminated $I(V)$ characteristics of the rectenna, we sweep over a range of values of $V_{O}$. The $v_{D}(t)$ and $i_{S}(t)$ cannot be solved directly for a given $V_{O}$ and $v_{S}(t)$ since the diode current is a function of $v_{D}(t)$. Therefore, for a given $V_{O}$, Equation (5) is solved iteratively to determine $v_{D}(t)$ and $i_{S}(t)$ using the diode dark $I(V)$ characteristics. The $v_{D}(t)$ is updated to a new value using Equation (5), and $i_{S}(t)$ is calculated again using the diode $I(V)$ characteristics. The procedure is repeated until the time-series sum of the difference between the nth and $(\mathrm{n}+1)$ th iterated values of $v_{D}(t)$ is less than a specific tolerance value, typically chosen to be a small fraction $(<0.001)$ of the maximum amplitude of $v_{S}(t)$, which is sufficient to achieve convergence.

To determine the relation between $i_{D}(t)$ and $v_{D}(t)$, we use either classical or PAT $^{22}$ theory. For classical largesignal analysis, the diode current is calculated using the diode dark $I(V)$ characteristics

$$
i_{D}(t)=I_{\text {dark }}\left[v_{D}(t)\right] .
$$

However, classical analysis of the rectenna does not take into account the quantization effect of the high energy photons on the electronic states in the diode, and is therefore appropriate only when ${ }^{25}$

$$
V_{D} \gg \hbar \omega / q \text {. }
$$

Here, the $V_{D}$ represents the amplitude of AC voltage across the diode. On the other hand, the quantum regime requires PAT theory and occurs at optical frequencies when

$$
V_{D} \ll \hbar \omega / q .
$$




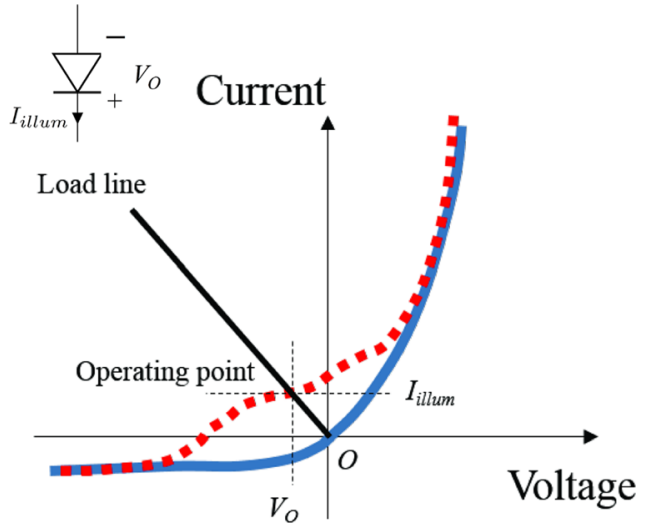

FIG. 3. Illustration of the illuminated $I(V)$ characteristics of the diode in a rectenna. The solid blue curve represents the dark $I(V)$ characteristics of the diode and the dotted red curve represents the illuminated $I(V)$ characteristics. The inset shows the sign of positive $I_{\text {illum }}$, corresponding to a diode dark current under positive bias, and the sign of $V_{O}$, which is negative. In this mode, the diode generates DC power in the second quadrant of the diode $I(V)$ characteristics. The load line intersects the illuminated $I(V)$ curve at the operating point, and the load resistance is chosen to maximize the power delivered to it.

In PAT theory, using the diode dark $I(V)$ characteristics and the diode voltage, the diode current is calculated as generalized by Tucker ${ }^{26}$

$i_{D}(t)=\iint d \omega^{\prime} W\left(\omega^{\prime}\right) I_{\text {dark }}\left(\omega^{\prime}+\frac{q V_{O}}{\hbar}\right) e^{-i \omega / t} d \omega^{\prime \prime} W\left(\omega^{\prime \prime}\right) e^{-i \omega^{\prime \prime} t}$.

Here, the integration is over the range of incident frequencies represented by $\omega$. The $W$ is the phase factor described in Ref. 26 and is the result of modulation of the Fermi level in the diode metal contact due to an applied time-dependent perturbation $v_{D}(t)$. The $W$ is related to the diode voltage through its Fourier transform

$$
\int_{-\infty}^{\infty} d \omega^{\prime} W\left(\omega^{\prime}\right) e^{-i \omega / t}=\exp \left\{-i \frac{q}{\hbar} \int^{t} d t^{\prime}\left[v_{D}\left(t^{\prime}\right)\right]\right\} .
$$

The average of $i_{D}(t)$ is the current $I_{\text {illum }}$ that flows through the load and results in a DC output power

$$
P_{\text {out }}^{D C}=\left|V_{O}\right| I_{\text {illum }}\left(V_{O}\right) .
$$

In the energy harvesting mode of the rectenna, the direction of $I_{\text {illum }}$ in the diode corresponds to that under positive bias. Since the diode generates DC power, $V_{O}$ is negative and power is produced where the plots of $V_{O}$ vs. $I_{\text {illum }}$ are in the second quadrant of the diode $I(V)$ characteristics, ${ }^{21}$ as illustrated in Figure 3. In Secs. IV and V, we use the procedure detailed above to calculate the optical response and the illuminated $I(V)$ characteristics of rectennas under monochromatic and broadband illumination.

\section{OPTICAL RESPONSE OF THE RECTENNA UNDER MONOCHROMATIC ILLUMINATION}

To demonstrate the method described above, we calculate the optical response of a rectenna under monochromatic illumination, with a diode having the piecewise linear $I(V)$ characteristics shown in Figure 4(a). The forward resistance of the piecewise linear diode is $50 \Omega$ and the reverse leakage current is zero. Such an $I(V)$ has a semi-classical "secant resistance" 9,21 of $100 \Omega$ at a $V_{O}$ of $0 \mathrm{~V}$, where the secant resistance is the reciprocal of the slope of the line connecting points on the $I(V)$ curve at $\pm \hbar \omega / q$ about $V_{O}$. This provides a match to an antenna impedance of $100 \Omega$ at $V_{O}=0 \mathrm{~V} .^{27}$ To contrast classical and PAT results we calculate the response of this rectenna for two different situations that represent Eqs. (7) and (8). For the purpose of defining the operating regime, since $V_{D}$ is a dynamic quantity and changes with $V_{O}$, for simplicity we will use $V_{S}$ as an approximation for $V_{D}$. For the piecewise linear diode used here, $V_{D}$ can vary from $V_{S} / 2$ to $V_{S}$ depending on the diode forward resistance and $V_{O}$, and therefore $V_{S}$ is a good estimate of $V_{D}$. We will show that PAT theory applies in both the situations, whereas classical theory applies only when $V_{D} \gg \hbar \omega / q$.

\section{A. Classical case: $V_{D} \gg h \omega / q$}

In the first case, corresponding to Equation (7), the photon energy is assumed to be $4 \mathrm{meV}$ and the input power is $200 \mu \mathrm{W}$, resulting in a relatively large $V_{S}$ of $0.4 \mathrm{~V}$ calculated using Equation (2). Knowing $v_{S}(t)$ and the diode $I(V)$ characteristics, we iteratively solve Equation (5) for different values of $V_{O}$ using both classical and PAT theories to calculate $v_{D}(t)$ and $i_{D}(t)$. The $P_{\text {out }}{ }^{D C}$ and the efficiency are calculated using Equations (11) and (1) and the plots of $I_{\text {illum }}$ and $\eta$ are shown in Figure 4(b). The $I_{\text {illum }}$ is maximum when the load is short circuited and decreases with an increase in $\left|V_{O}\right|$, approaching zero as $\left|V_{O}\right|$ exceeds the peak diode voltage amplitude. For the given conditions, the rectenna efficiency approaches a maximum of $\sim 40 \%$ at a $V_{O}$ of $-0.15 \mathrm{~V}$. The classical and PAT results overlap for $V_{D} \gg \hbar \omega / q$, verifying that classical analysis is valid.

In Figures 4(c) and 4(d), we plot the time-dependent source voltage, diode voltage, and diode current for operating voltages of $0 \mathrm{~V}$ and $-0.15 \mathrm{~V}$, respectively, to show the effect of $V_{O}$ on the rectenna response. In Figure 4(c), the peak value of the source voltage is $0.4 \mathrm{~V}$, and the $i_{D}(t)$ in the negative half of the AC cycle is zero, as is classically expected from the $I(V)$ characteristics. The average current over the full cycle gives $I_{\text {illum }}$ and is the rectenna shortcircuit current. Since $V_{O}=0 \mathrm{~V}$, the output power is zero and the rectenna functions as a detector rather than for energy harvesting.

In Figure 4(d), $V_{O}=-0.15 \mathrm{~V}$ and represents the case when a load is connected across the diode such that the rectenna efficiency is maximum. Because the voltage across the diode is clamped at $V_{O}$, in contrast with Figure 4(c) the diode voltage and current are positive over a smaller portion of the AC cycle. The $I_{\text {illum }}$ is positive, and the rectenna generates DC power. However, since the effective AC resistance of the piecewise linear diode shown in Figure 4(a) increases as $V_{O}$ becomes more negative, the result is a decrease in the power coupling efficiency between the antenna and the diode. ${ }^{27}$ Therefore, the overall efficiency is limited to $40 \%$ at $V_{O}=-0.15 \mathrm{~V}$. But this efficiency can be improved by 
(a)

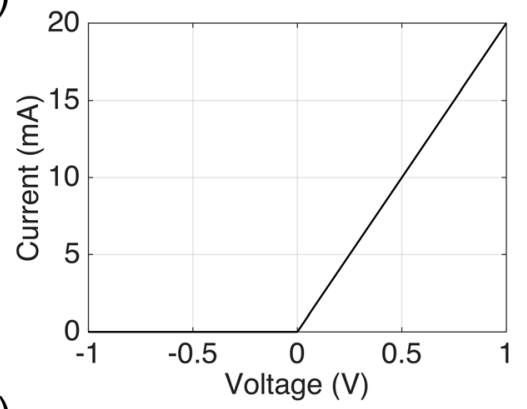

(c)
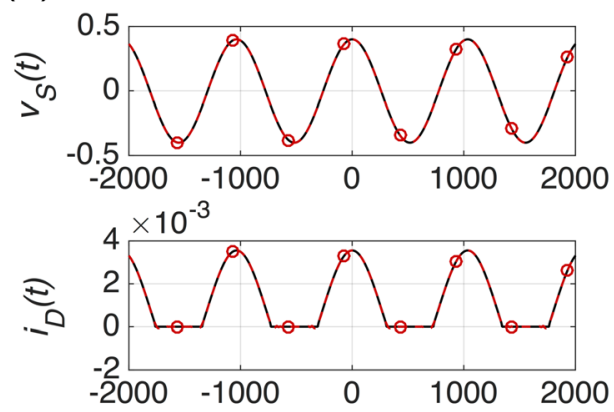

(d)
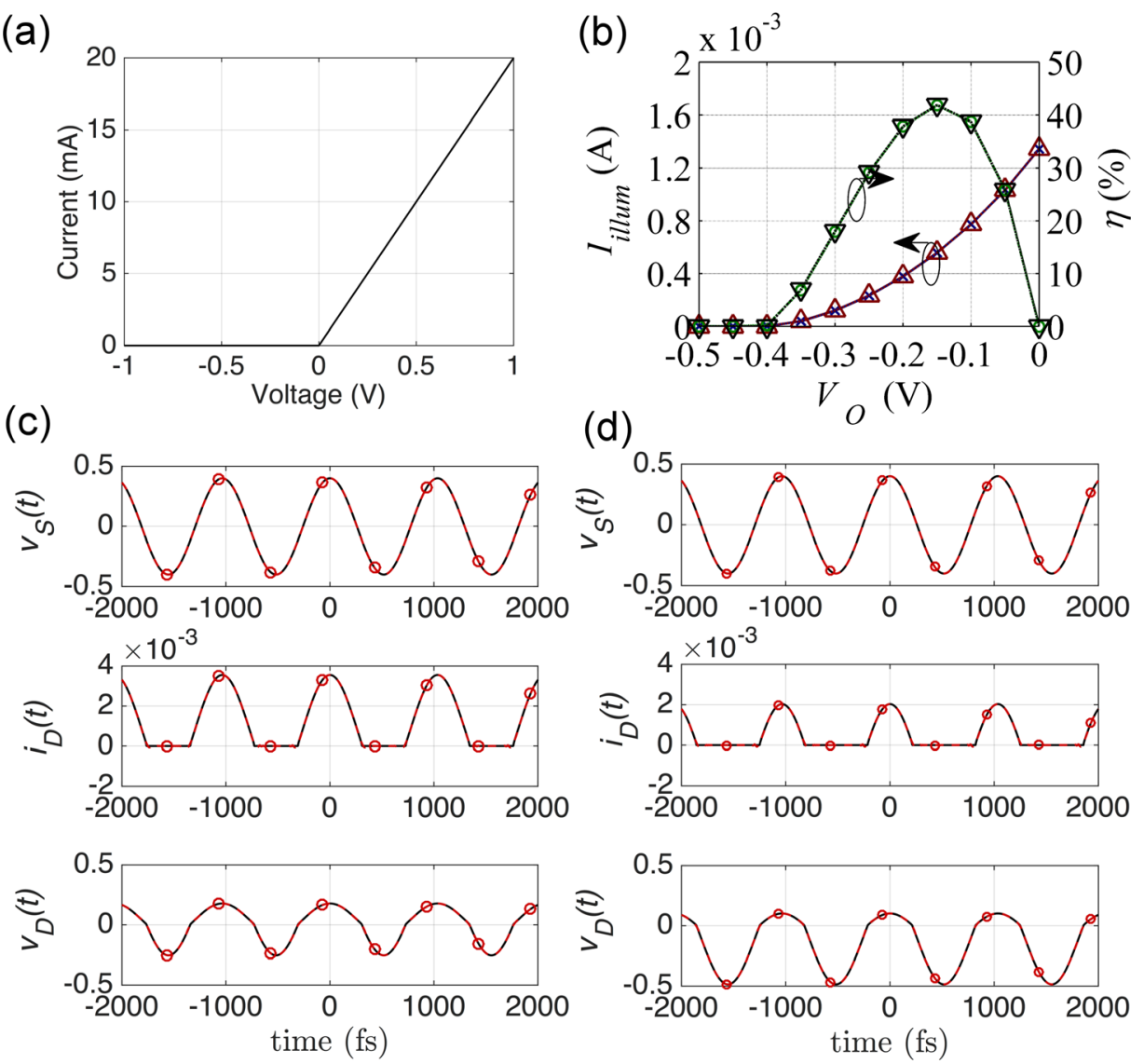

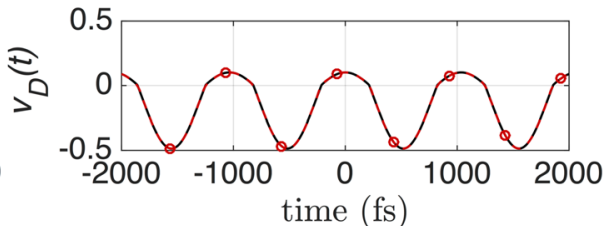

FIG. 4. Comparison of rectenna response to low-photon-energy illumination (4 meV, corresponding to $310 \mu \mathrm{m}$ ) using classical and photonassisted tunneling (PAT) theories at two operating voltages, for an input power of $200 \mu \mathrm{W}$. (a) The dark $I(V)$ characteristics of the piecewise linear diode having a forward resistance of $50 \Omega$ and zero reverse leakage current. (b) The PAT illuminated $I(V)$ (blue crosses) and efficiency (green circles) characteristics of the diode as a function of $V_{O}$. Also plotted are the classical illuminated $I(V)$ (red triangles) and efficiency (black flipped triangles) characteristics, which coincide with the PAT results. This efficiency is not the maximum efficiency of the rectenna, and can be improved using a diode $I(V)$ that matches well with the antenna at $\left|V_{O}\right|$ close to $V_{S}$. Plots of $v_{S}(t), i_{D}(t)$, and $v_{D}(t)$ were calculated using PAT theory (dotted red line) and classical theory (solid black) for (c) $V_{O}=0 \mathrm{~V}$ and (d) $V_{O}=-0.15 \mathrm{~V}$. The PAT and classical results superimpose under these conditions, and cannot be distinguished. adjusting the diode $I(V)$ characteristics to decrease the diode $\mathrm{AC}$ resistance and improve the coupling efficiency at the required operating point.

\section{B. Quantum case: $V_{D} \ll h \omega / q$}

The quantum operation occurs when rectennas are illuminated with low intensity and high frequency radiation. Such a case corresponds to Equation (8), and is important in the analysis of rectennas as solar cells because radiation from the sun consists of low intensity but high energy photons. Here, we calculate the rectenna response under the conditions, $\hbar \omega / q=2 \mathrm{~V}$ and $V_{S}=0.4 \mathrm{~V}$, using the method presented in Sec. III, and for the $I(V)$ characteristics shown in Figure 4(a).

The rectenna illuminated $I(V)$ and efficiency characteristics are shown in Figure 5(a). The rectenna has a quantized step-like response that shows up as a hump in the illuminated $I(V)$ characteristics. The $I_{\text {illum }}$ is maximum when the diode is short-circuited, i.e., $\left|V_{O}\right|=0$ and the antenna and the diode AC impedances are matched, which results in electrons absorbing single photons and giving $I_{\text {illum. As }}\left|V_{O}\right|$ increases, the AC resistance of the diode increases and the impedance match between the antenna and the diode is poor, resulting in reduced power transfer between them and a gradual decrease in the $I_{\text {illum }}$. When $\left|V_{O}\right|$ approaches $\hbar \omega / q$, the $I_{\text {illum }}$ reduces to zero, indicating that the photon energy is insufficient to assist electrons to tunnel through the diode and generate a current, in the same way as sub-bandgap energy photons do not contribute to the photocurrent in semiconductor solar cells. The resulting PAT maximum efficiency is limited to $\sim 48 \%$ at $V_{O}=-1.3 \mathrm{~V}$ due to the mismatch in the antenna and diode impedance. However, for an ideal diode with an impedance that matches the antenna impedance at $V_{O}=\hbar \omega / q$, the monochromatic efficiency in the quantum case $\left(V_{D} \ll \hbar \omega / q\right)$ can approach $100 \%$, as presented in Ref. 9.

Unlike the classical case, where the $I_{\text {illum }}$ in the illuminated $I(V)$ characteristics is expected to be non-zero up to a voltage of $\left|V_{O}\right| \sim V_{D}$, the $I_{\text {illum }}$ in the quantum case is nonzero at voltages greater than $V_{D}$ (up to $\left|V_{O}\right| \sim \hbar \omega / q$ ). In addition, the response calculated using classical theory, shown in Figure 5(b), does not exhibit the quantum humps expected in the quantum case of $V_{D} \ll \hbar \omega / q$. Therefore, classical theory gives incorrect results for the quantum case and cannot be used for calculating rectenna response in that range.

In Figures 5(c) and 5(d), we plot the $v_{D}(t)$ and $i_{D}(t)$, respectively, for $V_{O}=0 \mathrm{~V}$ and $V_{O}$ where the rectenna efficiency is maximum. In classical theory analysis, and as shown earlier in Figures 4(c) and 4(d), the current in the negative half of the AC cycle is zero. As a result, the time-dependent waveforms have a DC term, a fundamental term, and multiple harmonics. In contrast, for these examples, the PAT timedependent waveforms have only significant DC and fundamental terms, with harmonics that are small compared to the fundamental. This is characteristic of the quantum regime of diode operation and is due to the absence of higher-order photon absorption terms, limiting higher frequency currents and voltages. In Figure 5(d), the time domain plots are shown for $V_{O}=-1.3 \mathrm{~V}$ where the rectenna PAT efficiency is maximum, 
(a)

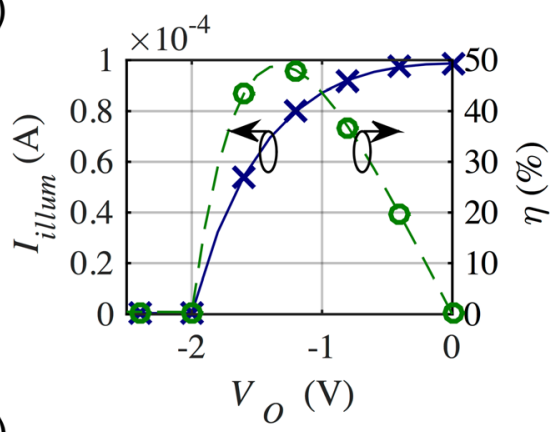

(c)
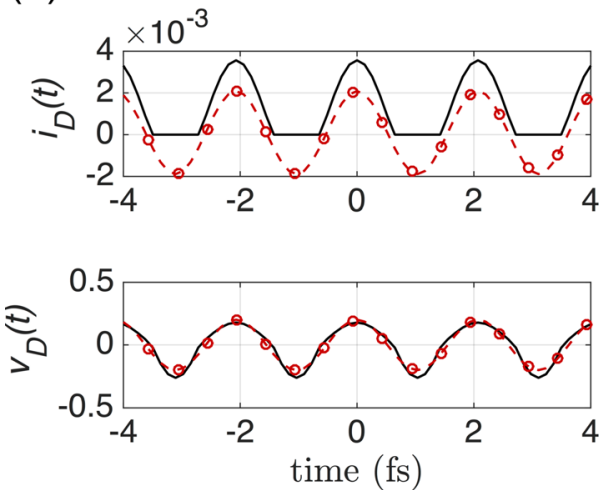

(b)

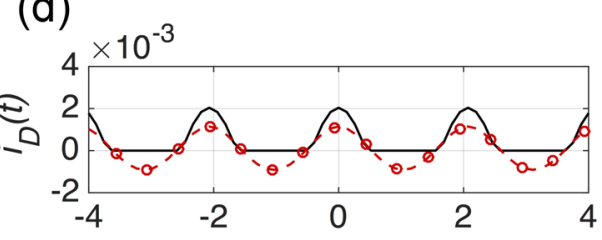

(d)

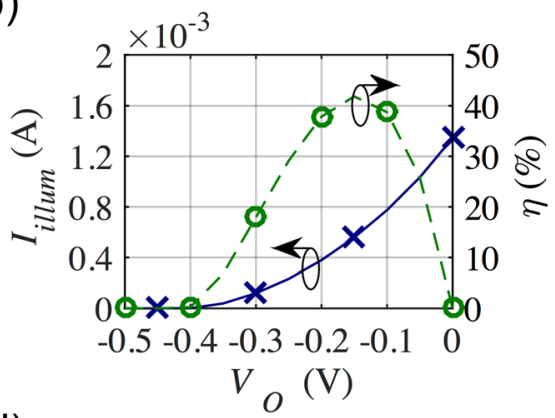

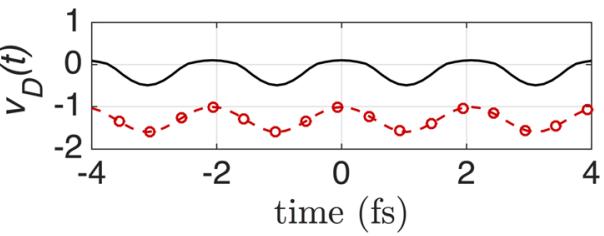

FIG. 5. Comparison of PAT and classical theory rectenna responses to high photon energy illumination ( $2 \mathrm{eV}$, corresponding to $620 \mathrm{~nm}$ ), at two operating voltages for an input power of $200 \mu \mathrm{W}$. The diode $I(V)$ characteristics are the same as in Figure 4(a). Illuminated $I(V)$ (blue crosses) and efficiency (green circles) characteristics calculated using: (a) PAT theory and (b) classical theory. Classical theory gives incorrect results for this quantum case where $V_{D} \ll \hbar \omega / q$. Also shown are plots of the time-dependent diode current $\left(i_{D}(t)\right)$ and diode voltage $\left(v_{D}(t)\right)$ calculated at $(\mathrm{c}) V_{O}=0 \mathrm{~V}$ using both PAT (red circles) and classical theories (solid black) and at (d) $V_{O}=-1.3 \mathrm{~V}$ for PAT theory and $V_{O}=-0.15 \mathrm{~V}$ for classical theory. The average of the diode currents in (d) gives the rectenna $I_{\text {illum }}$, shown in (a) and (b), and the chosen values for $V_{O}$ provide the maximum rectification efficiency. This efficiency is not the ultimate efficiency of the rectenna in the quantum case, and can be improved using a diode that matches the antenna impedance at $\left|V_{O}\right| \sim \hbar \omega / q$.

and $V_{O}=-0.15 \mathrm{~V}$ where the rectenna efficiency calculated using classical theory is maximum.

\section{OPTICAL RESPONSE UNDER BROADBAND ILLUMINATION: APPLICATION TO WASTE HEAT ENERGY HARVESTING}

The method shown above can be extended to determine the response of a rectenna to broadband sources, such as the sun or a hot blackbody. One potential application of rectennas is energy harvesting of waste heat from hot sources, ${ }^{28}$ which we consider below.

A hot source radiates a broad electromagnetic spectrum that can be collected by a rectenna using a broadband antenna. It has been shown that an ideal isotropic antenna that has a frequency-dependent effective area generates voltages with the spectral density of thermal noise across a hot resistor. $^{7,29}$ The frequency dependence of the antenna effective area may be included in the calculation by multiplying the effective area with the incident spectral density to determine the actual power at the antenna terminals. For simplicity, we assume that the impedance of the receiving antenna and its effective area is constant and independent of the frequency. A broadband antenna receiving this energy is represented by a source with a broad voltage spectrum whose shape may be approximated as the square root of the Planck blackbody spectrum. Using the inverse Fourier transform, we convert the frequency spectrum of the source voltage to the time domain. ${ }^{9}$ The phase distribution for the different frequency components is random and generated as normally distributed pseudorandom numbers. This randomly varying timedependent voltage is such that the power delivered to an impedance-matched load resistance is equal to the antenna input power. We use Equations (4) through (11) to determine the response of the rectenna to broadband illumination.

In Figure 6, we show the response of the diode to illumination from a hot body source of temperature $800 \mathrm{~K}$. The input power from the source at the antenna terminals is assumed to be $1 \mu \mathrm{W}$. The spectrum ranges from photon energies of $0.01 \mathrm{eV}$ to $4 \mathrm{eV}$ and peaks at $\sim 0.2 \mathrm{eV}$. We do not consider the effect of electron scattering relaxation time on rectenna performance at high frequencies, where it becomes relevant and could increase the power loss. The input power and the resulting source voltage is low such that the diode operates in the quantum regime, and as a result, it responds to each frequency component individually in the absence of higher order harmonics. As in a semiconductor solar cell, all photons with energy less than $q\left|V_{O}\right|$ do not tunnel through the diode and are unused, whereas photons with energy greater than $q\left|V_{O}\right|$ generate current at $V_{O}$ and are used only partially. Therefore, the efficiency of the rectenna peaks at $V_{O}=-0.12 \mathrm{~V}$ and is limited to $\sim 33 \%$.

In this paper, we concentrate on the method of analyzing the response rather than the ultimate efficiency of the rectenna and hence do not use a perfectly matched diode for the analysis. The theoretical efficiency can be improved by using a diode 


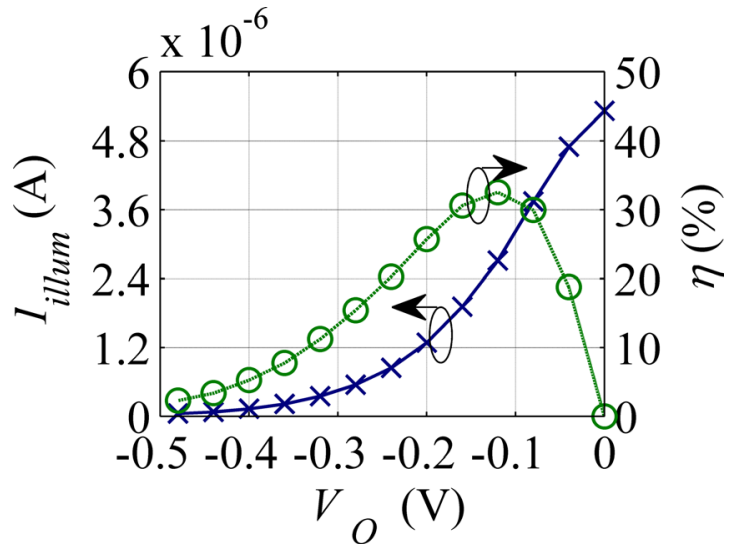

FIG. 6. Broadband illuminated $I(V)$ and efficiency characteristics of the diode in a rectenna calculated using PAT theory. The source is a blackbody of temperature $800 \mathrm{~K}$, the input power to the rectenna is $1 \mu \mathrm{W}$, and the diode $I(V)$ characteristics are the same as in Figure 4(a). The $I_{\text {illum }}$ (blue crosses) and efficiency (green circles) calculated using PAT theory are shown as a function of $V_{O}$. The maximum efficiency is $\sim 33 \%$ for this diode $I(V)$ and input conditions, and can be improved further using an $I(V)$ characteristic that matches the antenna impedance at a negative operating voltage.

$I(V)$ characteristic that matches well with the antenna impedance at the required $V_{O}$, as in Ref. 9. In practice, however, the diode will have a finite reverse leakage current, a finite capacitance, and a turn-on voltage greater than $0 \mathrm{~V}$. In addition, the power from the blackbody source will also be limited at the rectenna due to the finite coherence area of blackbody radiation at a nearby surface, ${ }^{30}$ and the frequency dependence of the effective area of the antenna. These constraints will result in a reduction in the efficiency of the rectenna.

\section{CONCLUSION}

In this paper, we described the procedure to calculate the illuminated $I(V)$ characteristics of rectennas. We analyzed the response of rectennas in the energy harvesting mode using a simple equivalent circuit, and solved for the time-dependent diode current and voltage using both PAT and classical theories. We used the diode $I(V)$ to calculate the illuminated $I(V)$ characteristics of the rectenna under monochromatic illumination and broadband illumination such as that from a hot body source. The monochromatic illumination results show that classical theory cannot be used to calculate the rectenna response when the photon energy is high and the flux is low, corresponding to $V_{D} \ll \hbar \omega / q$. Here an accurate calculation requires a PAT analysis, which incorporates the quantum operation of the rectenna through discrete steps in the illuminated $I(V)$ characteristics.

\section{ACKNOWLEDGMENTS}

This work was carried out under contracts from Abengoa Solar and RedWave Energy, Inc. The authors thank
Amina Belkadi, Bradley Pelz, and Shuai Yuan for helpful suggestions to improve the manuscript. The second author holds stock in RedWave Energy, Inc.

${ }^{1}$ J. G. Small, G. M. Elchinger, A. Javan, A. Sanchez, F. J. Bachner, and D. L. Smythe, Appl. Phys. Lett. 24, 275 (1974).

${ }^{2}$ A. Sanchez, C. F. Davis, K. C. Liu, and A. Javan, J. Appl. Phys. 49, 5270 (1978).

${ }^{3}$ M. Heiblum, S. Wang, J. Whinnery, and T. Gustafson, IEEE J. Quantum Electron. 14, 159 (1978).

${ }^{4}$ A. B. Hoofring, V. J. Kapoor, and W. Krawczonek, J. Appl. Phys. 66, 430 (1989).

${ }^{5}$ E. Briones, J. Alda, and F. J. González, Opt. Express 21, A412 (2013).

${ }^{6}$ B. J. Eliasson, Ph.D. thesis, University of Colorado, Boulder, 2001.

${ }^{7} \mathrm{~F}$. Yu, G. Moddel, and R. Corkish, in Advanced Concepts in Photovoltaics, edited by A. J. Nozik, G. Conibeer, and M. C. Beard, 1st ed. (Royal Society of Chemistry, 2014), pp. 506-546.

${ }^{8}$ G. Moddel and S. Grover, Rectenna Solar Cells, 2013 ed. (Springer, New York, 2013).

${ }^{9}$ S. Joshi and G. Moddel, Appl. Phys. Lett. 102, 083901 (2013).

${ }^{10}$ S. J. Byrnes, R. Blanchard, and F. Capasso, Proc. Natl. Acad. Sci. U. S. A. 111, 3927 (2014).

${ }^{11}$ G. A. E. Vandenbosch and Z. Ma, Nano Energy 1, 494 (2012).

${ }^{12}$ B. J. Eliasson and G. Moddel, U.S. patent 6,534,784 (18 March 2003).

${ }^{13}$ S. Grover, O. Dmitriyeva, M. J. Estes, and G. Moddel, IEEE Trans. Nanotechnol. 9, 716 (2010).

${ }^{14}$ P. Periasamy, H. L. Guthrey, A. I. Abdulagatov, P. F. Ndione, J. J. Berry, D. S. Ginley, S. M. George, P. A. Parilla, and R. P. O'Hayre, Adv. Mater. 25, 1301 (2013)

${ }^{15}$ K. Choi, F. Yesilkoy, G. Ryu, S. H. Cho, N. Goldsman, M. Dagenais, and M. Peckerar, IEEE Trans. Electron Devices 58, 3519 (2011).

${ }^{16}$ N. M. Miskovsky, P. H. Cutler, A. Mayer, B. L. Weiss, B. Willis, T. E. Sullivan, and P. B. Lerner, J. Nanotechnol. 2012, 1.

${ }^{17}$ I. Wilke, Y. Oppliger, W. Herrmann, and F. K. Kneubühl, Appl. Phys. A: Mater. Sci. Process. 58, 329 (1994).

${ }^{18}$ S. Krishnan, E. Stefanakos, and S. Bhansali, Thin Solid Films 516, 2244 (2008).

${ }^{19}$ G. Moddel, Z. Zhu, S. Grover, and S. Joshi, Solid State Commun. 152, 1842 (2012).

${ }^{20}$ Z. Zhu, S. Joshi, S. Grover, and G. Moddel, J. Phys. D: Appl. Phys. 46, 185101 (2013).

${ }^{21}$ S. Grover, S. Joshi, and G. Moddel, J. Phys. D: Appl. Phys. 46, 135106 (2013).

${ }^{22}$ P. K. Tien and J. P. Gordon, Phys. Rev. 129, 647 (1963).

${ }^{23}$ T.-W. Yoo and K. Chang, IEEE Trans. Microwave Theory Tech. 40, 1259 (1992).

${ }^{24}$ Note: In this paper, we update the voltage and current terminology from Refs. 9 and 21 to comply with conventional electric circuit terminology. We write the rectenna operating voltage as $V_{O}$, and use time-domain representation for the source current and voltage as $i_{S}(t)$ and $v_{S}(t)$, diode current and voltage as $i_{D}(t)$ and $v_{D}(t)$, respectively. In Ref. 9, the operating voltage was denoted as $V_{D}$, and the source voltage, diode current, and diode voltage were represented in the frequency domain as $\bar{V}_{S}, \bar{I}_{\omega}$, and $\bar{V}_{\omega}$, respectively.

${ }^{25}$ C. A. Hamilton and S. Shapiro, Phys. Rev. B 2, 4494 (1970).

${ }^{26}$ J. R. Tucker, IEEE J. Quantum Electron. 15, 1234 (1979).

${ }^{27}$ S. Joshi, S. Grover, and G. Moddel, in Rectenna Solar Cells, edited by G. Moddel and S. Grover (Springer, New York, 2013), pp. 47-67.

${ }^{28}$ G. Moddel, in Rectenna Solar Cells, edited by G. Moddel and S. Grover (Springer, New York, 2013), pp. 3-24.

${ }^{29}$ B. M. Oliver, Proc. IEEE 53, 436 (1965).

${ }^{30} \mathrm{H}$. Mashaal and J. M. Gordon, in Rectenna Solar Cells, edited by G. Moddel and S. Grover (Springer, New York, 2013), pp. 69-86. 